\title{
Wheat ear recognizing algorithm for high throughput wheat phenotyping
}

\author{
E. Komyshev ${ }^{1 *}$, M. Genaev ${ }^{1}$, S. Tumanyan ${ }^{1}$, N. Goncharov ${ }^{1,3}$, D. Afonnikov ${ }^{1,2}$, \\ V. Koval ${ }^{1}$ \\ ${ }^{1}$ Institute of Cytology and Genetics SB RAS, Novosibirsk, Russia \\ ${ }^{2}$ Novosibirsk State University, Novosibirsk, Russia \\ ${ }^{3}$ Novosibirsk State Agrarian University, Novosibirsk, Russia \\ *e-mail:komyshev@bionet.nsc.ru
}

Key words: spike, wheat ear, image processing, plant phenotyping

Motivation and Aim: Qualitative and quantitative analysis of morphological characteristics of wheat yield is an important step in breeding studies and developing new high-yielding varieties. A complex analysis of morphological features allows a more detailed understanding plant biology. The structure of the spike is one of the most important trait in cereals. Traditionally, spike analysis performed manually by experts. It includes the definition of the spike type, awnedness, dimensions, color, number of grains, etc. Defining, documenting, storing, and organizing a lot of different parameters manually is a time-consuming task. Therefore, it is important to develop computational tools for fast and efficient morphometry of wheat traits.

Methods and Algorithms: In this work, we present methods for spike phenotyping using image processing. We developed an algorithm for recognizing spike on the image, estimation of its characteristics and evaluate models based on it. The algorithm recognizes a color checker with a scale and wheat spike on a blue background, defines spike contour and performs its morphometry. Input images were obtained using two different protocols. The first protocol involved the horizontal placement of the ear on the surface without the use of any additional fixture. This approach is simpler, but it does not allow to obtain images of the ear in all projections. The second approach involved the vertical position of the ear with a special tripod. This makes it possible to obtain images of the ear in 4 projections.

Results: The extracted morphometric parameters are used to construct several shape recognition algorithms. The involved parameters include an ear profile, its length, width, area, color, awnedness etc. We compared the obtained algorithms to assess their applicability in problems of automated classification and clustering. The constructed models include parameters such as the profile of spike contour, the Fourier transform of the contour profile parameters, the trapezoidal model, the model based on the distances from the point of the center of mass to the points of the contour.

Conclusion: Wheat spike recognition algorithm implemented on the Java programming language. The OpenCV library for image processing was used [1] for algorithms implementation. Input program data is image of wheat spike located on a blue background. Developed wheat spike recognition algorithm can be used for highthroughput phenotyping of wheat spikes.

Acknowledgements: Supported by the RSF (No. 17-74-10148).

\section{References}

1. Bradski G., Kaehler A. (2008) Learning OpenCV: Computer vision with the OpenCV library. O’Reilly Media, Inc. 